\title{
A New Way to Build Multifacetted Ontologies for Elderly Care
}

\author{
Caroline Langensiepen \\ School of Science and \\ Technology \\ Nottingham Trent University \\ Clifton Lane, NG11 8NS, \\ Nottingham, United Kingdom
}

\author{
Ahmad Lotfi \\ School of Science and \\ Technology \\ Nottingham Trent University \\ Clifton Lane, NG11 8NS, \\ Nottingham, United Kingdom
}

\author{
Saisakul Chernbumroong \\ School of Science and \\ Technology \\ Nottingham Trent University \\ Clifton Lane, NG11 8NS, \\ Nottingham, United Kingdom
}

\author{
Pedro A. Moreno \\ Bioengineering and \\ Telemedicine Group \\ ETSI Telecommunication, \\ Technical University of Madrid \\ Madrid,28040, Spain
}

\author{
Enrique J. Gómez \\ Bioengineering and \\ Telemedicine Group \\ ETSI Telecommunication, \\ Technical University of Madrid \\ Madrid,28040, Spain
}

\begin{abstract}
The care of the elderly is complex, with multiple agencies and individuals involved. We propose a new way of developing an ontology to reflect these aspects within a real-time home monitoring system so that it captures real-life circumstances and interactions. Our new methodology incorporates iterative and evaluative stages to ensure the ontology captures implementable interactions and concepts. We have applied it to the iCarer project which was developed to assist informal carers with the activities of daily care for the elderly.
\end{abstract}

\section{Keywords}

Ontology, elderly care, dementia, assistive technology.

\section{Categories and Subject Descriptors}

E.2 [Data]: Data Storage Representations; I.6.1 [Simulation and Modeling]: Simulation Theory; J.3 [Life and Medical Sciences]: Health; J.4 [Social and Behavioral Sciences]: Psychology

\section{INTRODUCTION}

As the population ages, technology tries to fill the gaps by automating the monitoring and alarms needed, and by

\footnotetext{
*Ahmad Lotfi is the corresponding author. The corresponding email address is: ahmad.lotfi@ntu.ac.uk

Permission to make digital or hard copies of all or part of this work for personal or classroom use is granted without fee provided that copies are not made or distributed for profit or commercial advantage and that copies bear this notice and the full citation on the first page. Copyrights for components of this work owned by others than the author(s) must be honored. Abstracting with credit is permitted. To copy otherwise, or republish, to post on servers or to redistribute to lists, requires prior specific permission and/or a fee. Request permissions from permissions@acm.org.

PETRA '16, June 29-July 01, 2016, Corfu Island, Greece

(C) 2016 Copyright held by the owner/author(s). Publication rights licensed to ACM. ISBN 978-1-4503-4337-4/16/06 . .\$15.00

DOI: http://dx.doi.org/10.1145/2910674.2935831
}

providing support to the informal carers who are shouldering an increasing burden. The interaction of informal carers, health organisations, formal carers, and health care professionals with the elderly becomes ever more complex, leading to dangers of care aspects being missed, and informal carers becoming overwhelmed.

To that end, we propose key activities to ensure that the product of the methodology, the ontology, appropriately reflects the ecosystem of the elderly person's care in ways that can be recorded within a computer based system. These activities act as checks on the ontology breadth and depth, and may require us to remove interactions where they not be possible to implement.

The rest of the paper is organised as followed: Section 2 presents existing works related to the paper and research gap identification. Section 3 presents a novel method for constructing multi-faceted ontologies. Details of the data set used and experimental setup are presented in Section 4 which also discusses how it was applied to this situation. Finally, conclusions are presented in Section 5.

\section{RELATED WORK}

Ontologies have long been used to model information in the domain of healthcare. For example, Evchina et.al. present some related context-aware work on ontologies aimed at manage information in smart homes and health services [1]. Hussain et al [2] present a user model for recognising activities of daily living, and ACS-Ont is directed at the indoor and outdoor services of an AAL system [3]. Kuziemsky and Lau [4] point out that many of the difficulties associated with Healthcare Information Systems (HIS) relate to poor articulation of user needs and a complex environment. They question whether previous ontology development has concentrated on the completeness and quality of its content rather than its utility within a HIS. In comparison, for the medical ontology field, Hu et. al. [5] claim that requirements elicitation is a key stage to define the knowledge domain, and that feedback loops can be addressed to refine imperfectness 


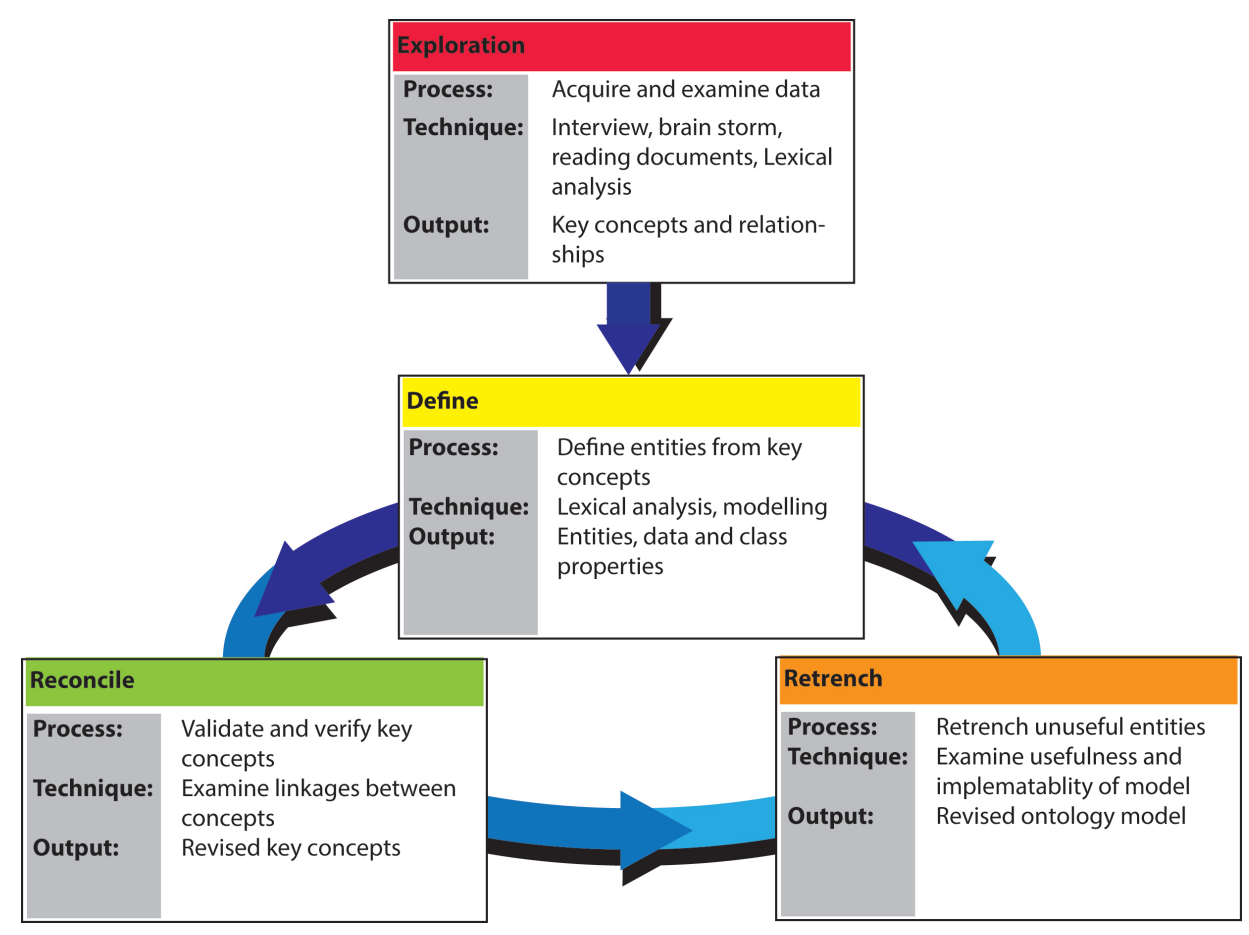

Figure 1: The proposed method for building multi-faceted ontologies: Explore-Define-Reconcile-Retrench (EDRR).

and enrich the core medical ontology with other relevant and necessary terms.

The key tasks of concept formation and semantic annotation of the knowledge domain in ontology engineering are time-consuming and requiring of considerable expertise [6]. It is thus important that the tasks are embedded in a wellformed process. Cristani [7] comments that most of the HIS methodologies follow a sequence of requirements acquisition, modelling concepts, and evaluation. Kuziemsky propose their own methodology which similarly follows these stages, but includes the development of the HIS as the vehicle for the evaluation. However there appears to be little iteration in the HIS methodologies described, whereas the discipline of software engineering as used for business systems has long accepted that the 'waterfall method' is not a cost-effective or timely methodology for incorporating changes to requirements that may be discovered during the development [8],[9].

\section{PROPOSED METHODOLOGY}

Our methodology is based on the premise of some key considerations that we believe are core to healthcare related systems:

1. That there are likely to be multiple core entities within the ontology, rather than the relatively simplistic ontologies that are expansions of attributes of a single concept,

2. That these entities generally represent both organisations and individuals (this also requires representing the hierarchical interaction of the individuals with the organisation)
3. Relationships may be polymorphic and dynamic ie they involve multiple entities rather than just binary relationships, and the nature of the relationships may change based on the states of the entities

4. The knowledge elicitation to generate the ontology needs to explicitly acknowledge its multisourced nature, in order to assist in the process of reconciling concepts which are expressed differently by different stakeholders.

In addition, when the ontology is incorporated directly into a HIS system, it has to be implementable - that is, the knowledge within it has to be capable of instantiation in software.

The proposed method consists of four key stages as shown in Figure 1: Explore, Define, Reconcile, and Retrench (EDRR).

- Explore - is the process by which information sources are acquired and examined. As in any knowledgebased process, the first stage is a wide ranging activity of identifying stakeholders, interviewing and document discovery and summarisation. The outputs of the explore phase will be a set of core concepts that can be traced back to the information sources. This traceability ensures that errors can be identified.

- Define - is the process whereby the relationships between the core concepts and to their subordinate concepts are specified. By subordinates we mean those aspects that only have meaning when considered in the context of the core entities. The subordinates are identified from the information sources by considering each of the core concepts in turn and addressing the environment around it, for related activities, entities and processes. 


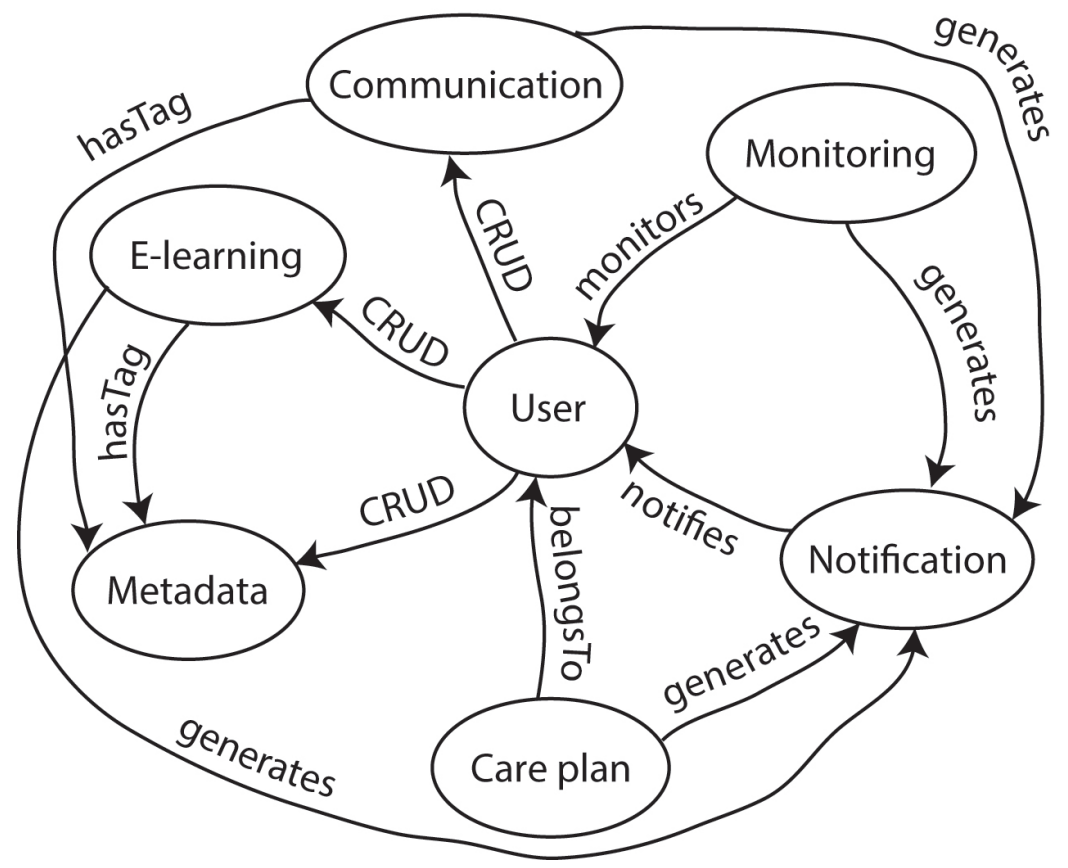

Figure 2: Initial capture of domain information from Exploration stage.

- Reconcile - is the process whereby the core concepts are brought back together and the links between them examined carefully, to ensure that there are no missing concepts - this may also give rise to the emergence of new concepts. An important aspect of this phase is examining situations where the links between the core concepts are not simply binary i.e. where multiple stakeholders and agencies are linked by some actions or processes. These transitive relationships are more difficult to capture but could be critical. Frequently, health and care services are criticised when vulnerable people 'fall through the cracks' and care is not appropriately transferred. This is an example of interactions involving multiple stakeholders to achieve a goal.

- Retrench - is the point at which we decide whether further iterations may be necessary. For the ontology to be of use within a system, the entities and relationships have to be capable of being instantiated and populated with dynamic data so that inferences might be drawn. For example, a concept called 'hygiene' for an 'elderly person', mediated via a 'carer' is not well defined.So we either go round the cycle again and define hygiene more explicitly (eg into washing, bathing, teeth cleaning) or remove it from our system. If there is no way that these can be populated with instance data due to lack of sensors, the retrenchment could result in a decision that we would remove the unimplementable areas and stop the process.

\section{APPLICATION OF METHODOLOGY}

This section presents the details of the data sets and experimental setup used to evaluate the proposed EDRR, and discussion of insights achieved by using the methodology.

\subsection{Data Sets}

We used the data collected in the iCarer project [10], from stakeholders involved in elderly care i.e. older adults, informal carers, and care organizations. Brainstorming sessions with social care support staff and interviews with informal carers in various care situations were carried out. The informal carers' characteristics and the technological feasibility were taken into account when identifying users' needs.

The first stage in EDRR is exploration where data sets, information and knowledge are examined, extracted, and analyzed for potential keywords and linkages. In order to illustrate the processes, parts of the interview data below are quoted:

"Managing own care tasks and the tasks and visits of external services and professional caregivers that take care of the elderly can be overwhelming for some informal caregivers. An easy to use planning tool for care tasks can make this more easy for the informal caregiver."

"Many informal caregivers have trouble sleeping due to excessive $24 / 7$ care tasks, stress or worries about the situation. Keeping good track on the sleep patterns and stress levels will enable a tool to give advice to the informal caregiver on how he can improve his sleeping pattern and coop with stress or worries. This will increase the health and quality of life of the informal caregiver which will in turn lead to better care capabilities of the informal caregiver."

"Informal caregivers often treat their elderly based on their own previous experience and skills, not from professional education or care experience. Care for the elderly could improve greatly 
Key concepts
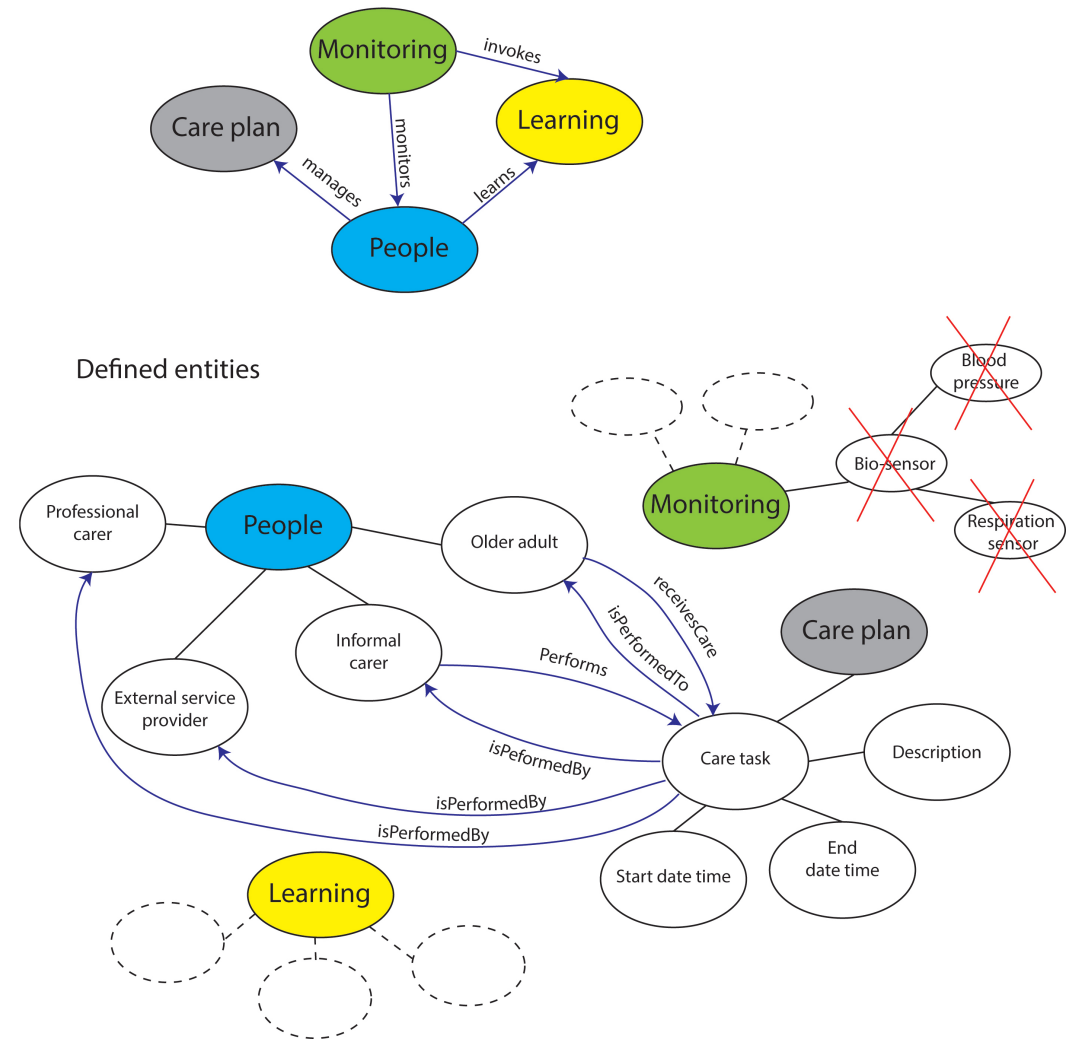

Figure 3: Diagram to illustrate some key concepts and defined entities.

if the informal caregiver receives sufficient feedback and education on how they should perform their care tasks."

\subsection{Exemplar Situation}

John is an older adult who lives at home with his wife Jane whom is his main carer. He can carry out most of his daily activities normally, but requires some assistance with grocery shopping, meals preparation, and bathing. Jane helps with those activities except for grocery shopping which is performed by their son, Joe. Joe shops and delivers the grocery to his parents every Saturday. Jane wants a system that allows her to monitor her husband, and also enables her to see all the care he receives. Joe wants a system to see what care task he has to provide. Jane also thinks it is a good idea if she can communicate with other informal carers and sends useful care topic/lessons. A formal carer from city council wants to be able to assign care tasks to informal carers to maintain care quality in the home.

\subsection{Using EDRR}

As a result of the information gathered during the Exploration stage, many concepts were identified including people, care plan, learning, and monitoring. This initial understanding of the domain is shown in Figure 2. Terms and relationships were then extracted from available data sets related to these concepts. For example, extracted terms related to the 'people' concept are professional carer, informal carer, older adult, and external provider. To help to assess the information gathered, exemplar situations were created, and used to test the concepts and entities developed through the methodology

In the Define stage, the core concepts were expanded with relevant entities. Figure 3 illustrates how the outline of the domain was expanded by this addition. During the execution of the Define stage, several intermediary entities were identified. The intermediary entity refers to the entity which mediates between two entities. In a normal sentence with a structure of subject-verb-object, an ontology can be easily modelled using classes and object properties terminologies. For example, "A carer can care for one or more older adults" can be modelled as Carer caresFor OlderAdult. However, with an intermediary entity, a sentence is complex and modelling is not as straightforward. Examples involving intermediary entities are:

"A formal carer assigns a care task to an informal carer."

"An informal carer sends a care topic to another informal carer."

In order to model these intermediary entities, we follow three steps:

1. identify the intermediary entity,

2. construct a single sentence for the intermediary entity by using the intermediary entity as the main subject,

3. model each single sentence generated in step 2 . 
A)

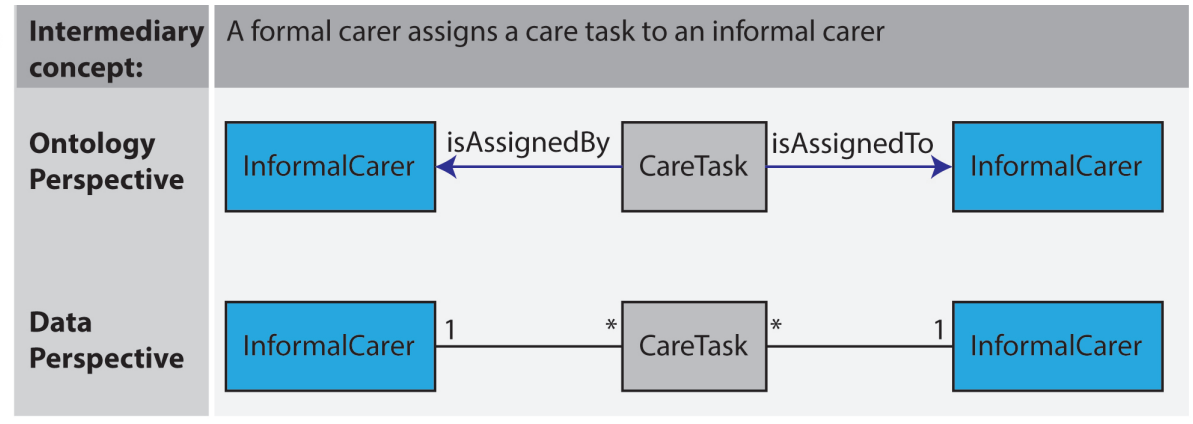

B)

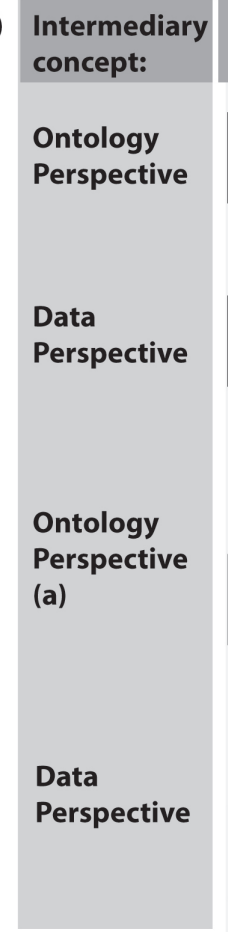

An informal carer sends a care topic to another informal carer

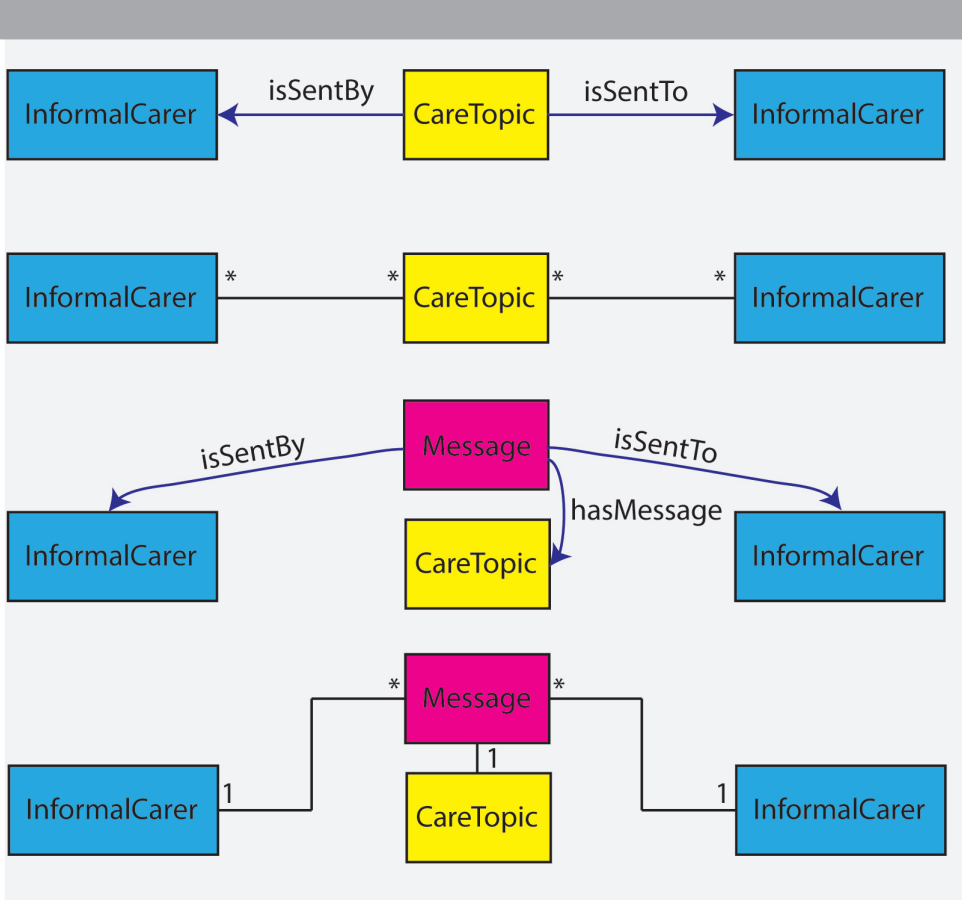

Figure 4: Modelling intermediary concepts: ontology versus data perspectives.

For example, using the first sentence from the above statements, the intermediary entity is CareTask. Using aforementioned technique, the single sentences are "A care task is assigned by a formal carer" and "A care task is assigned to an informal carer". These sentences can be modelled using three classes: CareTask, FormalCarer, InformalCarer, and the care task has object properties: isAssignedBy and is AssignedTo (See Figure 4A). Considering the second sentence in the intermediary entity examples, one can easily mistake a care topic as an intermediary entity. If the care topic is used as the intermediary entity, the resulted model will not be able to cope when more than one carer sends the same care topic to other carers. It will not be possible to distinguish who sends the topic to whom. Therefore, a new intermediary entity needs to be created. If we look at the data perspective of the intermediary entity using the care topic as the intermediary entity (Figure 4B), it can be seen that the relationships are many-to-many. This indicates that an additional term is required to mediate between these entities. This evaluation of the relationships formed part of the Reconcile stage, which ensured that the entities and terms could be appropriately related.

The Retrench stage is used to ensure that the process is suitably realistic. For example, the 'Monitoring' concept was originally expanded with entities such as blood pressure, respiratory rate etc.. These were not applicable in the intended application due to technology limitations. Such entities are removed during the retrench stage to make sure that the remaining entities were relevant to the targeted application and could be populated with live data for inferencing.

As a result of applying the methodology, the ontology was built using Protégé software. Figure 5 shows a small aspect of the ontology as developed, populated by simulated data instances relating to the case study, to evaluate its fitness for purpose. As seen in this fragment, the care tasks such as shopping (which would be specified as part of the care plan) are associated with the informal carer and the elderly person, though they may be allocated to multiple carers depending on the circumstances as discussed above. The detailed analysis and design of the relationships between these concepts in the ontology that has occurred as a result of our application of EDRR ensures that we can correctly represent these 


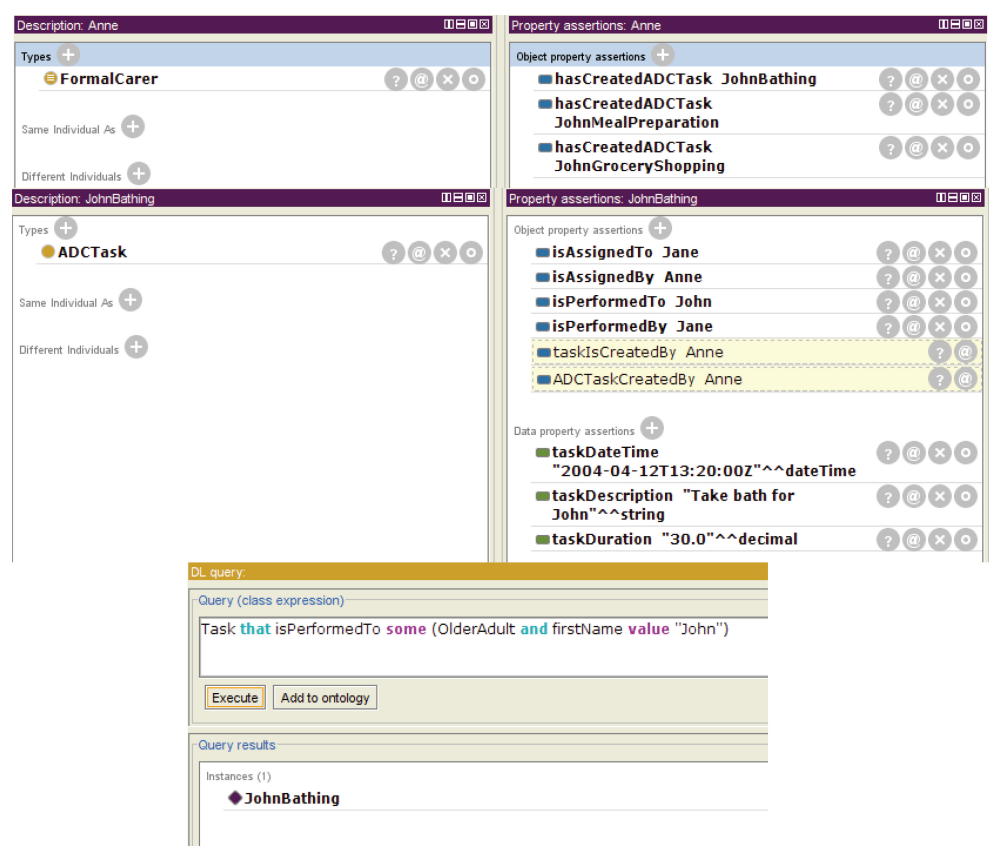

Figure 5: Sample of Protégé implementation of ontology.

and allocate data instances and events appropriately.

\section{CONCLUSION}

Developing an assistive living system involved very different stakeholders such as older adults, carers, policy makers, service providers with different needs and expectations. It is important that systems for this area can fully take account of these differences, and catch the potential misinterpretations before they get embedded into the technology. The example discussed in Section 4.3 shows how the proposed techniques assist in solving potential problems from multiple stakeholders and intermediary concepts. The reconcile stage requires key concepts to be rechecked which can help identify new concepts or linkages. The iteration of the stages allow the ontology to be debugged to make sure it only contains terms that are useful in the live system. We have found that using a systematic and formalised process, with explicit iteration, allowed the identification of potential problems and also gave confidence that the ontology could be successfully populated with data from the users. Further work will use the system and data instantiated within its nodes to draw inferences about activities and problems.

\section{ACKNOWLEDGEMENT}

The iCarer project has received funding and support through Innovate UK and the Active and Assisted Living (AAL) joint European programme under the Grant number AAL-20125-239 (iCarer).

\section{REFERENCES}

[1] Evchina, Yulia and Dvoryanchikova, Aleksandra and Lastra, José L. Martinez, Ontological framework of context-aware and reasoning middleware for smart homes with health and social services, IEEE Int. Conf. on Systems, Man, and Cybernetics (SMC) (2012) 985-990.
[2] Hossain, Md Aynal and Ahmed, Dewan T., Virtual caregiver: an ambient-aware elderly monitoring system, IEEE Trans. on Information Technology in Biomedicine 16 (6) (2012) 1024-1031.

[3] Bernardos, Ana M. and del Socorro Bernardos, M. and Iglesias, Josué and Casar, José R., Data Modeling for Ambient Home Care Systems,Distributed Computing and Artificial Intelligence (2010) 333-340.

[4] C. E. Kuziemsky, F. Lau, A four stage approach for ontology-based health information system design, Artificial Intelligence in Medicine 50 (3) (2010) 133-148.

[5] $\mathrm{Hu}, \mathrm{Bo}$ and Dasmahapatra, Srinandan and Dupplaw, David and Lewis, Paul and Shadbolt, Nigel, Reflections on a medical ontology, International journal of human-computer studies 65 (7) (2007) 569-582.

[6] Lim, Soon Chong Johnson, Liu, Ying, Lee, Wing Bun, A methodology for building a semantically annotated multi-faceted ontology for product family modelling, Advanced Engineering Informatics 25 (2) (2011) 147-161.

[7] M. Cristani, R. Cuel, A Survey on Ontology Creation Methodologies., Int. J. Semantic Web Inf. Syst. 1 (2) (2005) 49-69.

[8] G. Booch, Object-oriented analysis and design with applications, Addison-Wesley, 1991.

[9] B. Boehm, A Spiral Model of Software Development and Enhancement, SIGSOFT Softw. Eng. Notes11, (4) (1986), 14-24.

[10] iCarer - Intelligent Care Guidance and Learning Services Platform [ONLINE] Available at: http://icarer-project.eu/. [Accessed 20 January 2016]. 